\title{
Genome-wide association study of white-coat effect in hypertensive patients
}

Jenni M Rimpelä ${ }^{\mathrm{a}}$, Teemu Niiranen ${ }^{\mathrm{b}}$, Antti Jula ${ }^{\mathrm{c}}$, Ilkka H Pörsti ${ }^{\mathrm{d}}$, Antti Tikkakoski ${ }^{\mathrm{e}}$, Aki Havulinna ${ }^{\mathrm{f}}$, Terho Lehtimäki ${ }^{\mathrm{g}}$, Veikko Salomaa ${ }^{\mathrm{h}}$, Kimmo K Kontula ${ }^{\mathrm{i}}$, Timo P Hiltunen $^{\mathrm{j} *}$

${ }^{a}$ Research Program for Clinical and Molecular Metabolism, Faculty of Medicine, University of Helsinki; Department of Medicine, University of Helsinki and Helsinki University Hospital, Helsinki, Finland; Adr: 00290 Helsinki, FINLAND; Tel: +358 9 4711; Email: jenni.rimpela@helsinki.fi; ${ }^{b}$ Department of Public Health Solutions, National Institute for Health and Welfare, Helsinki, Finland; Department of Medicine, University of Turku and Turku University Hospital, Turku, Finland, Adr: 20521 Turku, FINLAND; Tel: +358 2313 0000; Email: teemu.niiranen@utu.fi; ${ }^{c}$ Department of Public Health Solutions, National Institute for Health and Welfare, Helsinki, Finland; Adr: 00271 Helsinki, FINLAND; Tel: +358 29524 6000; Email: antti.jula@thl.fi; ${ }^{d}$ Faculty of Medicine and Life Sciences, University of Tampere and Tampere University Hospital, Tampere, Finland; Adr: 33521 Tampere, FINLAND; Tel: +358 3311 611; Email: ilkka.porsti@uta.fi; ${ }^{e}$ Department of Clinical Physiology and Nuclear Medicine, Tampere University Hospital, Tampere, Finland; Adr: 33014 Tampere, FINLAND; Tel: +358 3311 611; Email: antti.tikkakoski@pshp.fi; ${ }^{f}$ Institute for Molecular Medicine Finland, FIMM, University of Helsinki, Helsinki, Finland; Adr: 00014 Helsingin yliopisto, FINLAND; Tel +358 2941 911, Email: aki.havulinna@thl.fi; ${ }^{g}$ Department of Clinical Chemistry, Fimlab Laboratories and Finnish Cardiovascular Research Center Tampere, Faculty of Medicine and Life Sciences, University of Tampere, Tampere, Finland; Adr: 33014 Tampereen Yliopisto, FINLAND; Tel +358 3355111 , Email: terho.lehtimaki@uta.fi; ${ }^{h}$ Department of Health, National Institute for Health and Welfare, Helsinki, Finland; Adr: 00271 Helsinki, FINLAND; Tel: +358 29524 6000; Email: veikko.salomaa@thl.fi; ${ }^{i}$ Research Program for Clinical and Molecular Metabolism, Faculty of Medicine, University of Helsinki; Department of Medicine, University of Helsinki and Helsinki University Hospital, Helsinki, Finland; Adr: 00290 Helsinki, FINLAND; Tel: +358 9 4711; Email: kimmo.kontula@helsinki.fi; ${ }^{j}$ Research Program for Clinical and Molecular Metabolism, Faculty of Medicine, University of Helsinki; Department of Medicine, University of Helsinki and Helsinki University Hospital, Helsinki Finland; Adr: 00290 Helsinki, FINLAND; Tel: +358 9 4711; Email: timo.hiltunen@hus.fi 


\section{Genome-wide association study of white-coat effect in hypertensive patients}

Background: White-coat effect (WCE) confounds diagnosis and treatment of hypertension. The prevalence of white-coat hypertension is higher in Europe and Asia compared to other continents suggesting that genetic factors could play a role.

Methods: To study genetic variation affecting WCE, we conducted a two-stage genome-wide association study involving 1343 Finnish subjects. For the discovery stage, we used Genetics of Drug Responsiveness in Essential Hypertension (GENRES) cohort $(n=206)$, providing the mean WCE values from up to four separate office/ambulatory recordings conducted on placebo. Associations with $p$ values $<1 \times 10^{-5}$ were included in the replication step in three independent cohorts: Haemodynamics in Primary and Secondary Hypertension (DYNAMIC) ( $n=182)$, Finn-Home study $(n=773)$ and Dietary, Lifestyle and Genetic Determinants of Obesity and Metabolic Syndrome (DILGOM) $(n=182)$.

Results: No single nucleotide polymorphisms reached genome-wide significance for association with either systolic or diastolic WCE. However, two loci provided suggestive evidence for association. A known coronary artery disease risk locus rs2292954 in SPG7 associated with systolic WCE (discovery $p$ value $=2.2 \times 10^{-6}$, replication $p$ value $=0.03$ in Finn-Home, meta-analysis $p$ value $2.6 \times 10^{-4}$ ), and rs 10033652 in RASGEF1B with diastolic WCE $\left(\right.$ discovery $p$ value $=4.9 \times 10^{-6}$, replication $p$ value $=0.04$ in DILGOM, meta-analysis $p$ value $\left.=5.0 \times 10^{-3}\right)$.

Conclusion: This study provides evidence for two novel candidate genes, SPG7 and RASGEF1B, associating with WCE. Our results need to be validated in even larger studies carried out in other populations.

Keywords: white-coat effect, hypertension, gene, genome-wide, $S P G 7, R A S G E F 1 B$

\section{Introduction}

A proper diagnosis and rational treatment of hypertension, the leading risk factor for cardiovascular disease, is confounded by the white-coat effect (WCE), implying that blood pressure (BP) measured by healthcare personnel at the office (OBP) is usually higher than BP measured using ambulatory $\mathrm{BP}(\mathrm{ABP})$ monitoring or by the patients themselves at home [1,2]. WCE is perceived as a pressor response driven by an anxious reaction to BP measurement [3]. It is a common phenomenon that 
becomes more pronounced at higher BP levels [4]. The main problem caused by WCE is white-coat hypertension, identified by elevated OBP, but normal ABP or home BP [1]. At present, the consensus is that white-coat hypertension and accentuated WCE carry little cardiovascular risk compared to true normotension [5], but there is a growing body of contradictory reports of association with target organ damage [6], cardiovascular events [7] and increased cardiovascular mortality [8].

Despite the obvious problems it causes for clinicians in decision of using antihypertensive treatment, the mechanisms underlying WCE are not well understood. However, many correlates for WCE have been identified. A recent meta-analysis of existing literature (70 studies, 86,167 patients) identified sex, body mass index (BMI), smoking status and systolic BP (SBP) level as the most important predictors of WCE [9]. Interestingly, the ARTEMIS Study, an international multicenter study involving 14,143 patients from 27 countries, reported higher prevalence of white-coat hypertension in Europe and Asia compared to Australia, America and Africa, suggesting that genetic factors could partly explain WCE [10]. Apart from a few candidate gene studies, the genetic background of WCE has not been extensively studied. Recently, a Japanese group conducted a genome-wide association study (GWAS) of WCE, using a discovery study with 295 and replication study with 236 individuals, but no significant associations were discovered [11], possibly due to only moderate reproducibility of WCE [12]. Thus, even larger studies of WCE genetics are required, also to provide new insights for genetics of BP regulation [13]._In addition, identifying genetic loci associated with WCE would provide a tool to investigate the causal relationship between white coat hypertension and cardiovascular target organ damage through Mendelian randomization.

To study possible genetic influence on WCE, we conducted a GWAS of WCE in the Finnish Genetics of Drug Responsiveness in Essential Hypertension (GENRES) cohort $(n=206)[14,15]$. As GENRES includes repeated measurements of OBP and ABP under placebo treatment, it provides a 
unique opportunity to reduce intraindividual variability of WCE. Replication analyses were conducted in three other Finnish cohorts: Hemodynamics in Primary and Secondary Hypertension (DYNAMIC) $(n=182)$ [16,17], the Finn-Home study $(n=773)$ [5,18] and Dietary, Lifestyle and Genetic Determinants of Obesity and Metabolic Syndrome (DILGOM) ( $n=182)$ [19].

\section{Methods}

\section{Overview of participating studies}

Four Finnish study cohorts (combined $n=1343$ ) were included in this study: GENRES, DYNAMIC, Finn-Home and DILGOM.

GENRES cohort originates from a randomized, double-blind, cross-over, placebo-controlled study on pharmacogenomics of drug responsiveness in essential hypertension. The clinical phase was conducted between 1999 and 2004 in Helsinki University Hospital (clinicaltrials.gov NCT03276598). The study design and rationale have been previously published elsewhere $[14,15]$. In brief, the subjects were moderately hypertensive Finnish men (aged 35 to 60 years) who went through four one-month drug treatment periods (losartan $50 \mathrm{mg}$, amlodipine $5 \mathrm{mg}$, hydrochlorothiazide $25 \mathrm{mg}$ and bisoprolol $5 \mathrm{mg}$ once a day) in randomized order, separated by onemonth placebo periods. OBP and 24-hour ABP measurements were carried out at the end of each period (Supplementary Figure 1). The inclusion of intervening placebo periods in the study design provides a unique opportunity to study WCE as a mean of up to four separate measurements conducted at two-month intervals. Thus, GENRES cohort was used for the discovery GWAS. To further reduce within-subject variability, only the 206 unrelated subjects with $\geq 3$ placebo WCE measurements and genotyping data available were included in the present study. Each subject gave written informed consent and the study was approved by the Ethics Committee of Helsinki University Central Hospital and National Agency of Medicines of Finland. 
DYNAMIC (clinicaltrials.gov NCT01742702) is an ongoing study to investigate hemodynamic changes in primary and secondary hypertension with a non-invasive hemodynamic measurement protocol $[16,17]$. A total of 187 subjects with WCE assessment available were genotyped. One subject was excluded due to low success rates (>95\%) of genotyping, one subject was excluded due to morbid obesity $(\mathrm{BMI}>40)$ and three subjects were excluded due to first degree relativeness; ultimately, 182 unrelated subjects were included in the present replication study. Each subject gave written informed consent and the study was approved by the Ethics Committee of Tampere University Hospital.

Finn-Home cohort originates from a multidisciplinary epidemiological survey, the Health 2000 Survey, carried out in Finland from 2000 to 2001, from which individuals aged 45-74 years with home BP monitors available were recruited to the study [5,18]. This population sample underwent office and home BP measurements and risk factor evaluation to investigate the prognostic significance of home BP. A total of 786 subjects had BP and genotype data available. Twelve subjects were excluded due to morbid obesity (BMI > 40) and one subject due to WCE residual differing more than four standard deviations from mean, resulting in 773 unrelated subjects to be included in the present replication study. The study protocol of the Health 2000 survey was approved by Epidemiology Ethics Committee of Helsinki and Uusimaa Hospital region and all participants gave written informed consent.

The DILGOM (Dietary, Lifestyle and Genetic determinants of Obesity and Metabolic syndrome) was a population survey conducted in 2007 to assess the effects of environmental and genetic factors on obesity and metabolic syndrome. Four hundred and ninety-four subjects from Southwestern Finland participated in a cardiovascular substudy. In 2014 the still living participants of the cardiovascular substudy were invited to a study comparing traditional and novel BP measurements [19]. Both WCE and genetic data were available for 209 subjects. Four morbidly obese $(\mathrm{BMI}>40)$ subjects and 23 subjects over the age of 75 were excluded resulting in 182 
unrelated subjects to be included in the present replication cohort. Each subject gave written informed consent and the study was approved by the Ethics Committee of the Hospital District of Southwest Finland.

\section{BP measurement in participating studies}

In GENRES OBP was measured after a 30-minute rest using a semi-automated oscillometric device (Omron M4; Omron Healthcare, Tokyo, Japan) by a study nurse. Three measurements were taken and the mean of the last two was used for the analyses. 24-hour ABP measurement was conducted with a device equipped with a position sensor (Diasys Integra; Novacor, Rueil-Malmaison, France). Readings were taken every 15 minutes when standing and every 30 minutes when supine. Intense physical activity was not permitted. Daytime was defined as hours between 7 am and $10 \mathrm{pm}$. Single observations were excluded from the analysis due to low pulse pressure $(<15 \mathrm{mmHg}$ if SBP $<120$ and $<20 \mathrm{mmHg}$ if SBP $>120$ ), high heart rates $\geq 110$, lying down during daytime or high physical activity. For a recording to be accepted for this study, $>15$ daytime measurements were required.

In DYNAMIC OBP was measured by a physician after at least 10 minutes of rest in the seated position using a calibrated aneroid sphygmomanometer (Heine Gamma G7, HEINE Optotechnik, Herrsching, Germany). The phase I and V Korotkoff sounds were used to identify systolic and diastolic BP, respectively. Two measurements at about 2-minute intervals were taken and the mean of these values was used for the analyses. If either systolic or diastolic readings differed by more than $10 \mathrm{mmHg}$, a third measurement was taken and the mean value of the last two measurements was used for the analyses. Heart rate was recorded by pulse palpation after the last measurement in the sitting position. Microlife WatchBP O3 monitor (Microlife AG, Widnau, Switzerland) was used to measure 24-hour ABP. Readings were taken every 20 minutes during the day (7 am to $10 \mathrm{pm})$. Single observations were excluded using the same criteria as described for GENRES. 
In Finn-Home OBP was measured by a trained nurse with a conventional calibrated mercury sphygmomanometer. SBP and diastolic BP (DBP) were defined according to Korotkoff sounds I and IV. OBP was determined as the mean of two measurements performed at 2-minute interval after 10-minute rest. Home BP was self-measured with an automatic oscillometric device (Omron model HEM-722C, Omron Corporation, Tokyo, Japan). Study participants took duplicate selfmeasurements every morning (between 6 am and 9 am) and every evening (between $18 \mathrm{pm}$ and 21 pm) on seven consecutive days in sitting position with approximately 2-minute interval. Home BP was determined as the mean of 14 duplicate measurements (28 measurements).

In DILGOM OBP was measured twice by a nurse after a 3-minute rest at 1-minute intervals with an oscillometric BP monitor (Microlife WatchBP Office Central, Microlife AG, Widnau, Switzerland). Twenty-four-hour ABP was then recorded with a Microlife WatchBP O3 monitor (Microlife AG, Widnau, Switzerland). Readings were taken every 20 minutes during the day (from 7 am to $10 \mathrm{pm}$ ) and every 30 minutes during the night (from $10 \mathrm{pm}$ to $7 \mathrm{am}$ ). Daytime BP was defined as the mean of all other BP values except the actual sleeping period at night.

\section{Genotyping and quality control}

The genotyping methods and quality control steps for GENRES have been described in detail before [15]. The DNA samples of the GENRES study subjects were genotyped at the Institute for Molecular Medicine Finland (FIMM, Helsinki, Finland) using the Illumina HumanOmniExpress BeadChip (Illumina, San Diego, CA, USA). Initial quality control steps included overall genotyping success rate (exclusion if $<95 \%$ ), identity-by-state clustering and gender check. Of the 709,357 genotyped autosomal SNPs, 707,658 passed these quality control steps. Afterwards, SNPs with

genotyping success rate $<95 \%$ (759 SNPs), Hardy-Weinberg equilibrium $p$ value $<1 \times 10^{-5}(256$ SNPs) and minor allele frequency $<0.01$ (77,231 SNPs) were excluded resulting in 629,414 genotyped autosomal SNPs available for the analysis. Even though we used only the genotyped SNPs for the GWAS, imputed SNPs were used to test candidate SNPs derived from a previous 
GWAS of WCE (11). For the latter purpose, GENRES genotypes were imputed using IMPUTE2 [20] and the Hapmap2 CEU release 22 reference panel after pre-phasing with ShapeITv2. Lastly, the effect of population stratification on WCE was tested with principal components (PCs) generated with program Smartpca from Eigensoft package [21]. A reduced dataset of 114,487 SNPs with $r^{2}<0.5$ and the long-range linkage disequilibrium (LD) regions excluded [22] were used. Three significant PCs for population stratification with $p$ values $<0.10$ were identified with program Twstats from Eigensoft package, but they were not associated with either systolic or diastolic WCE residuals (all $p$ values > 0.20) and accordingly were not included in GWAS analysis.

The DNA samples of the DYNAMIC study subjects were genotyped at the Institute for Molecular Medicine Finland (Helsinki, Finland) using the Illumina HumanOmniExpress BeadChip (Illumina, San Diego, CA, USA). One subject was excluded due to low success rate $(<95 \%)$. The 11 SNPs used for replication passed the same quality control steps as described for GENRES above.

The genotype data of the Finn-Home cohort originating from the Health 2000 survey was derived from previously conducted GWASs. The DNA samples were genotyped using Illumina Infinium HD Human610-Quad BeadChip (Illumina, San Diego, CA, USA). Subsequent imputation of SNP genotype data was carried out with MACH 1.0.16 using the HapMap3 CEU and TSI populations as an imputation reference. If the selected SNP was not genotyped, the imputed value was used. Imputed genotypes were used for SNPs rs9614176, rs10965522, rs2650673 and rs10033652.

The genotype data of the DILGOM study subjects was derived from previously conducted GWASs. The DNA samples were genotyped with Illumina Cardio-MetaboChip and Illumina 610K arrays (Illumina, San Diego, CA, USA) at the Wellcome Trust Sanger Institute (Cambridge, UK) and at the Institute for Molecular Medicine Finland (Helsinki, Finland). Unobserved SNPs were imputed using 1000 Genomes haplotypes Phase I integrated variant set release (ShapeITv2) in 
NCBI build 37 version June 2014. If the selected SNP was not genotyped, the imputed value was used. Imputed genotypes were used for SNPs rs10965522, rs2650673 and rs2053425.

\section{Calculation of white-coat effect residuals for genome-wide association analysis}

WCE was calculated as the difference between OBP and daytime ABP in GENRES, DYNAMIC and DILGOM. In the Finn-Home cohort, the WCE was calculated as the difference between OBP and home BP. The two WCE traits, systolic WCE and diastolic WCE, were analyzed separately as continuous variables using IBM SPSS Statistics 22.0 (IBM Corp., Armonk, NY) and were approximately normally distributed. Before genetic association analyses we adjusted both systolic and diastolic WCE for significant covariates $(p<0.10$ for either systolic or diastolic WCE) using linear regression (Supplementary Table 1). The included covariates were age, BMI, corresponding daytime ABP and smoking status. Other covariates tested but not included were waist-hip ratio, use of antihypertensive drugs before entry to the study, serum creatinine level, urinary excretion of sodium after the first placebo period and three PCs (see above). For the replication cohorts that included women, sex was included as a covariate as well as the current use of BP medication where appropriate.

\section{Genome-wide association analysis}

\section{Discovery phase}

The discovery GWAS was performed in the GENRES cohort, using covariate-adjusted WCE residuals and linear regression under additive genetic model in PLINK v1.07 [23]. The results were plotted using Manhattan and quantile-quantile (Q-Q) plots generated with QQman package in R. Regional Manhattan plots were generated with Locuszoom package in R. Bonferroni-corrected $p$ value $<5 \times 10^{-8}$ was considered genome-wide significant, and, based on the hypothesis-generating nature of the present study, $p$ value $<1 \times 10^{-5}$ was considered suggestively significant. 


\section{Replication phase}

Replication analyses in three independent cohorts were carried out for the statistically most significant SNPs from each locus with $p$ value $<1 \times 10^{-5}$. For the replication analyses, covariateadjusted WCE residuals were generated using linear regression and same covariates (plus sex) as in the discovery GWAS. A Bonferroni-corrected $p$ value $<0.0015$ [0.05 / (11 SNPs $\times 3$ studies)] and same direction of effect was considered as successful replication, while a $p$ value $<0.05$ and same direction of effect was considered suggestively significant. We tested for SNP $\times$ sex interaction in the replication cohorts and conducted a separate analysis in men if it was significant $(p<0.10)$. We also conducted meta-analysis of the top WCE SNPs in all available studies using inverse-variance model with fixed effects in METAL (24). We defined significant results as $p$ values $<5 \times 10^{-8}$ and $p$ values $<1 \times 10^{-5}$ were considered to represent a suggestive association.

\section{Replication of candidate variants from a previous genome-wide association study of white- coat effect}

We conducted a replication analysis for the associations found in a previous GWAS of WCE [11]. SNPs with $p$ values $<5 \times 10^{-5}$ were chosen for replication, with the exclusion of three SNPs that were monoallelic in Finns. Thus, nine SNPs were analyzed for systolic WCE and eight SNPs for diastolic WCE. Bonferroni-corrected $p$ value $<0.003$ (0.05 / 17 SNPs) with the same direction of effect was considered as a significant replication.

\section{Functional analysis of top associations in silico}

We used Variant Effect Predictor (VEP) to characterize the leading variant from each locus with $p$ value $<1 \times 10^{-5}[25,26]$. Variants characterized as intergenic SNPs were annotated to the closest gene using Ensembl browser version 92 [27,28]. We further conducted an expression quantitative trait locus (eQTL) analysis using the Genotype-Tissue Expression (GTEx) Portal [29,30] to identify whether the leading variants were cis-eQTLs for nearby genes. 
As WCE is an anxious reaction to having BP measured [3], we looked if any of the leading WCE SNPs had cross-trait associations at a nominal $p$ value $<0.05$ with major personality traits including neuroticism in three large GWASs with publicly available results: the UK Biobank

GWAS by Neale lab $(n=\sim 300,000)$ [31], the Genetics of Personality Consortium (GPC) GWAS ( $n$ $=\sim 63,000)[32]$ and the Social Science Genetic Association Consortium (SSGAC) GWAS $(n=$ $\sim 180,000-300,000$ ) [33]. We also looked if the leading WCE SNPs had previously been associated at a nominal $p$ value $<0.05$ with BP or pulse rate in large BP GWASs with publicly available data: the International Consortium of Blood Pressure Genome-Wide Association Studies (ICBP) GWAS ( $n=\sim 63,000)$ [34] and UK Biobank GWAS by Neale lab $(n=\sim 300,000)$ [31]. The GWAS results were queried using the Phenoscanner database [35,36] and MR-Base PheWAS database $[37,38]$.

\section{Results}

\section{General comparison of the study samples}

The characteristics of the discovery cohort (GENRES) and the replication cohorts (DYNAMIC, the Finn-Home study and DILGOM) are summarized in Table 1. The discovery GWAS was carried out in GENRES, which consisted of men only, but all replication cohorts consisted of both men and women. Thus, a separate replication analysis was performed for men, if sex $\times$ SNP interaction was significant $(p<0.10)$. The participants of GENRES and DYNAMIC were all hypertensive, whereas the Finn-Home study and DILGOM were population-based studies comprising both normotensive and hypertensive individuals. The mean systolic WCE ranged from 1.2 to $8.0 \mathrm{mmHg}$ and the mean diastolic WCE from 0.3 to $4.0 \mathrm{mmHg}$. The standard deviations of the WCEs were the smallest in GENRES (Table 1).

\section{Genome-wide association study of systolic white-coat effect}

The Q-Q plot from the discovery GWAS of systolic WCE showed little evidence of genomic 
inflation and supported the idea that some of the associations may be significant (Supplementary Figure 2A). Manhattan plot of the results (Figure 1A) illustrates four loci with $p<1 \times 10^{-5}$, but no SNPs with genome-wide significance $\left(p<5 \times 10^{-8}\right)$ were identified. The four loci with $p<1 \times 10^{-5}$ in the discovery GWAS are presented in Table 2, and the local Manhattan plots are depicted in Supplementary Figure 3A-D. As the GENRES subjects received four different antihypertensive monotherapies in a rotational fashion separated by one-month placebo periods (Supplementary Figure 1), it was pertinent to test if the top associations derived from the placebo periods were similar when WCE data during administration of the four different drugs were analyzed. The effects of the SNPs were consistently in the same direction as during placebo periods and $p$ values for the associations often remained statistically significant (Supplementary Table 2). In the GENRES study, the WCEs during the drug treatment periods did not differ statistically significantly from each other, nor from the mean WCE during placebo periods.

The most significant association in the discovery GWAS was displayed by rs9614176 on chromosome $22\left(\beta=5.3 \mathrm{mmHg}, p=8.7 \times 10^{-7}\right)$ near EFCAB6. In the replication analysis, however, we did not find support for this locus. No SNP reached the Bonferroni-corrected $p$ value $(0.003)$ in the replication analysis, but rs2292954 ( $\beta=6.2 \mathrm{mmHg}, p=8.7 \times 10^{-6}$ in GENRES), a missense mutation of $S P G 7$ gene, showed a suggestive replication in the Finn-Home study $(n=773, \beta=2.4$ $\mathrm{mmHg}, p=0.03$ ). The effect was in the same direction in DYNAMIC, but the association was not significant $(\beta=2.2 \mathrm{mmHg}, p=0.4)$; however, in DILGOM the effect was in the opposite direction $(\beta=-3.5 \mathrm{mmHg}, p=0.05)$. Meta-analysis of all four cohorts did not yield more significant $p$ values (Supplementary Table 3). Sex $\times$ SNP interaction was significant only for rs4454147 in the FinnHome study, and the $p$ value for the association of systolic WCE with this SNP was stronger in men $(\beta=-1.6 \mathrm{mmHg}, p=0.07)$ than in men and women combined $(\beta=-0.2 \mathrm{mmHg}, p=0.8)$ (Table 3).

\section{Genome-wide association study of diastolic white-coat effect}

The Q-Q plot from the discovery GWAS of diastolic WCE showed little evidence of genomic 
inflation, suggesting that some of the associations may be significant (Supplementary Figure 2B).

The Manhattan plot (Figure 1B) shows seven loci with $p<1 \times 10^{-5}$, but no genome-wide significant SNPs were identified. The results of the discovery GWAS are summarized in in Table 4 and Supplementary Figure 4A-G. Like for systolic WCE, we also tested if the top diastolic WCE associations derived from the placebo periods were similar when WCE data during the four different drug periods were analyzed. As shown in the Supplementary Table 2, all the associations were consistently in the same direction and mostly statistically significant.

In the replication analysis, no SNP reached the Bonferroni-corrected $p$ value (0.003). However, rs10033652, an intronic SNP in RASGEF1B showed suggestive replication in DILGOM $(\beta=1.8 \mathrm{mmHg}, p=0.04)$ (Table 4). In the meta-analysis of all four cohorts no SNP reached genome-wide significance (Supplementary Table 3). Two SNPs (rs2650673 and rs11062980) had $p$ values $<0.10$ for sex $\times$ SNP interaction in at least one of the replication cohorts (Table 5). When analyzed separately, men showed stronger association for rs11062980 with diastolic WCE in $\operatorname{DILGOM}(\beta=1.9 \mathrm{mmHg}, p=0.07)$ than men and women combined $(\beta=-0.6 \mathrm{mmHg}, p=0.5)$.

\section{Candidate variants from a previous genome-wide association study of white-coat effect}

We conducted a replication analysis for the associations found in a previous GWAS of WCE [11]. No SNP reached the Bonferroni-corrected $p$ value $<0.003$ (Supplementary Table 4). Lowest $p$ values were 0.03 for rs314668 (with different direction of effect) and 0.06 for rs 7717374 with the same direction of effect, both for diastolic WCE.

\section{Discussion}

The present study was aimed at identifying genetic loci associated with WCE, i.e. the magnitude of the BP response to a clinic visit, using a systematic GWAS approach (discovery phase, replication studies and meta-analysis). Altogether, our study involved 1343 Finnish subjects, indicating the largest sample to study genetic associations of this trait thus far. We did not identify genome-wide 
significant SNPs for either systolic or diastolic WCE, but 11 loci were identified at suggestive $p$ value $<1 \times 10^{-5}$, four for systolic WCE and seven for diastolic WCE. In the replication analysis, some support was obtained for two of these loci, rs2292954 in SPG7 and rs10033652 in RASGEF1B. In addition, two other SNPs (rs4454147 and rs11062980) with previous nominal associations with BP traits [31,34], showed stronger association with WCE in men than in men and women combined, pointing to a possible sex-specific effect. Lastly, cross-trait analysis revealed that four out of the 11 WCE loci identified in the hypertensive GENRES cohort have been previously nominally associated with neuroticism-related traits in at least two large publicly available GWASs of these phenotypes [31-33].

The most interesting finding from this study is the association of rs 2292954 in SPG7 with larger systolic WCE as it has previously been associated with increased risk for coronary artery disease $\left(p=2.3 \times 10^{-5}\right)$ in the large CARDIoGRAM GWAS study [39]. It was also nominally associated with SBP $(p=0.01)$ and pulse rate $(p=0.02)$ in the recent UK Biobank GWAS by Neale lab [31], supporting its role in BP regulation. rs2292954 is a missense variant in the SPG7 gene causing the substitution of alanine for threonine, which is predicted to be tolerated by SIFT and benign by Polyphen (Supplementary Table 5). SPG7 codes for paraplegin, a subunit of mitochondrial mAAA protease, which is a pleiotropic enzyme regulating many mitochondrial functions [40]. As shown in the local Manhattan plot (Supplementary Figure 3B), this locus contains multiple linked SNPs $\left(r^{2}>0.8\right)$ with low $p$ values, such as rs 12960 (for the effect allele A: systolic WCE $\beta=6.1 \mathrm{mmHg}, p=4.0 \times 10^{-6}, r^{2}=0.98$ with rs 2292954 in GENRES). rs 12960 is likewise a missense variant of SPG7, causing an amino acid change from arginine 688 to glutamine which is predicted by Polyphen to be probably damaging. The functional characterization of rs 12960 suggests that this allele is a gain-of-function variant of $S P G 7$ causing an increased activity of SPG7-containing mAAA proteases and resulting in increased reactive oxygen production in the mitochondria [41]. eQTL analysis of GTEx database [29,30] identified both rs2292950 and rs12960 
as cis-acting eQTLs for SPG7, but also for nearby RPL13 (ribosomal protein L13) (Supplementary Table 5).

We also observed association of rs 10033652 with a larger diastolic WCE. We did not find any evidence that this intronic SNP of RASGEF1B or any other SNP in LD (Supplementary Figure 4E) could be functional. RASGEF1B codes for a guanine-nucleotide exchange factor (GEF). Its expression in macrophages is stimulated by contact with bacterial lipopolysaccharides and it controls diverse Ras-protein-mediated signaling networks [42]. A recent study found that RasGEF $1 B$ circular RNA controls the expression of ICAM-1, an important adhesion molecule expressed in the endothelium, which attracts white blood cells to the vessel wall [43].

As the GENRES study consisted of only men, we investigated possible sex $\times$ SNP interaction in the replication cohorts. For two SNPs, rs4454147 and rs11062980, sex seemed to interact with the effect of the SNP on WCE. We observed a stronger association of rs4454147 with systolic WCE in men than in men and women combined. This intergenic SNP does not seem to be functional (Supplementary Table 5), but it has been nominally associated with BP levels previously in the ICBP GWAS ( $p=0.02$ for SBP and $p=0.03$ for DBP) [34] and UK Biobank GWAS by Neale lab ( $p=0.001$ for SBP and $p=0.06$ for DBP) [31] (Supplementary Table 6). Interestingly, Phenoscanner $[35,36]$ query revealed that the associations of this SNP and obesity-related traits were also stronger in men than in women in the GIANT study [44] suggesting a possible sexspecific effect. Likewise, rs 11062980 had a stronger association with diastolic WCE in men in DILGOM compared to men and women combined. This intergenic SNP was a weak eQTL for a nearby lincRNA gene RP11-320N7.2 and pseudogene HSPA8P5 in GTEx database [29,30]. It has previously been nominally associated with DBP in UK Biobank GWAS by Neale lab $(p=0.03)$ [31] and notably also with nervous feelings ( $p=0.03$ in UK Biobank GWAS by Neale lab) [31] and neuroticism ( $p=0.006$ in the SSGAC GWAS) [33] (Supplementary Table 7). Possible sex- 
dependent genetic associations, although rarely investigated in BP genetics, would not be unexpected, as sex differences in BP regulation are well known [16].

We also sought to replicate the findings from a previous GWAS of WCE in the Japanese HOMED-BP study [11]. None of the associations replicated at the stringent Bonferroni-corrected $p$ value limit $<0.003$. The best $p$ values were 0.03 for rs314668 (closest gene ACTBL2) with different direction of effect and 0.06 for rs7717347 (strong eQTL for STRN4 expression in the GTEx database $[29,30])$ with same direction of effect. In addition, both WCE associated SNPs were also nominally associated with BP readings and neuroticism-related traits in the UK Biobank GWAS by Neale lab [31] (Supplementary Table 7).

Psychological mechanisms related to anxiety are thought to influence WCE $[3,45]$. There is also some evidence that personality traits are associated with white-coat hypertension [46]. However, these associations were significant only in a subgroup that perceived themselves as hypertensive $[45,46]$. Thus, the association of anxious personality to WCE might depend on the self-experienced hypertension status of the patient. As all GENRES subjects were hypertensive, we queried three large publicly available GWASs of psychological traits (GPC, SSGAC, UK Biobank GWAS) [31-33] for association of the top WCE SNPs from GENRES GWAS with neuroticism and related psychological traits. Four out of eleven WCE loci, including rs9614176, rs7606893, rs2650673 and rs11062980 showed nominal association with neuroticism-related traits in at least two of the large GWASs queried (Supplementary Tables 6 and 7). Further studies on these loci could elucidate the mechanisms of cardiovascular response to stress in hypertensive patients.

The present study has several limitations. The very specific phenotype under study necessarily limited possibilities to collect extensive patient materials, and accordingly the sample size is an obvious limitation of our study. On the other hand, the main strength of our study is the use of repeated measurements of WCE both in the discovery cohort (GENRES) and in the largest replication cohort (Finn-Home). Secondly, the discovery cohort (GENRES) consisted of men only, 
whereas all three replication cohorts included both men and women; indeed, sex has been shown to associate with WCE [9]. On the other hand, this enabled us to study possible sex-dependent associations. Third, while GENRES and one of the replication samples (DYNAMIC) were dominated by hypertensive individuals, the other two replication cohorts (Finn-Home and DILGOM) were derived from population surveys. This may have caused a selection bias in GENRES. In addition, in GENRES, DYNAMIC and DILGOM the WCE was defined as the difference between OBP and daytime ABP, whereas in Finn-Home the WCE was defined as the difference between OBP and home BP.

\section{Conclusions}

We identified two novel candidate genes for white-coat effect in human hypertension. It is of note that some of the functions of these two genes are related to cardiovascular pathophysiology, mitochondrial function and inflammation. We also found some evidence for sex-dependent genetic associations with white-coat effect. Cross-trait association analysis gave some support for the hypothesis that the hypertensive white-coat effect could be associated with neuroticism-related personality traits. Additional studies from other populations, however, are required to validate our data.

\section{Acknowledgements}

We thank Ms. Susanna Saarinen for excellent technical help and Reeta Kulmala, RN and Paula Erkkilä, RN for invaluable contribution to the hemodynamic recordings of the DYNAMIC study.

\section{Disclosure of interest}

Authors declare no conflict of interest. 


\section{Funding}

The GENRES Study was supported by grants from the Sigrid Jusélius Foundation and the Finnish

Foundation for Cardiovascular Research. The DYNAMIC study was supported by the Finnish Foundation for Cardiovascular Research, Sigrid Jusélius Foundation, and Päivikki and Sakari Sohlberg Foundation. 


\section{References}

[1] Pickering TG, James GD, Boddie C, et al. How common is white coat hypertension? JAMA 1988;259(2):225-228.

[2] Stergiou GS, Zourbaki AS, Skeva Il, et al. White coat effect detected using self-monitoring of blood pressure at home: comparison with ambulatory blood pressure. Am J Hypertens 1998;11(7):820-827.

[3] Grassi G, Turri C, Vailati S, et al. Muscle and skin sympathetic nerve traffic during the "whitecoat" effect. Circulation 1999;100(3),222-225.

[4] Myers MG. The white-coat effect in treated hypertension. Blood Press Monit 1996;1(3),247249.

[5] Hanninen MR, Niiranen TJ, Puukka PJ, et al. Prognostic significance of masked and white-coat hypertension in the general population: the Finn-Home Study. J Hypertens 2012;30(4):705712.

[6] Sung SH, Cheng HM, Wang KL, et al. White coat hypertension is more risky than prehypertension: important role of arterial wave reflections. Hypertension 2013;61(6):13461353.

[7] Tientcheu D, Ayers C, Das SR, et al. Target organ complications and cardiovascular events associated with masked hypertension and white-coat hypertension: analysis from the Dallas Heart Study. J Am Coll Cardiol 2015;66(20):2159-2169.

[8] Mancia G, Facchetti R, Bombelli M, et al. Long-term risk of mortality associated with selective and combined elevation in office, home, and ambulatory blood pressure. Hypertension 2006;47(5):846-853.

[9] Sheppard JP, Fletcher B, Gill P, et al. Predictors of the home-clinic blood pressure difference: a systematic review and meta-analysis. Am J Hypertens 2016;29(5):614-625.

[10] Omboni S, Aristizabal D, De la Sierra A, et al. Hypertension types defined by clinic and ambulatory blood pressure in 14143 patients referred to hypertension clinics worldwide. Data from the ARTEMIS study. J Hypertens 2016; 34(11):2187-2198.

[11] Ogata S, Kamide K, Asayama K, et al. Genome-wide association study for white coat effect in Japanese middle-aged to elderly people: The HOMED-BP study. Clin Exp Hypertens 2018;40(4):363-369.

[12] Elijovich F, Laffer CL. Magnitude, reproducibility, and components of the pressor response to the clinic. Hypertension 1990;15(2 Suppl):I161-I615. 
[13] Padmanablhan S, Caulfield M, Dominczak AF. Genetic and molecular aspects of hypertension. Circ Res 2015;116(6):937-959.

[14] Hiltunen TP, Suonsyrjä T, Hannila-Handelberg T, et al. Predictors of antihypertensive drug responses: initial data from a placebo-controlled, randomized, cross-over study with four antihypertensive drugs (The GENRES Study). Am J Hypertens 2007;20(3):311-318.

[15] Hiltunen TP, Donner KM, Sarin AP, et al. Pharmacogenomics of hypertension: a genomewide, placebo-controlled cross-over study, using four classes of antihypertensive drugs. $\mathbf{J}$ Am Heart Assoc 2015;4(1):e001521.

[16] Kangas P, Tahvanainen A, Tikkakoski A, et al. Increased cardiac workload in the upright posture in men: Noninvasive hemodynamics in men versus women. J Am Heart Assoc 2016;5(6):e002883

[17] Tikkakoski AJ, Tahvanainen AM, Leskinen MH, et al. Hemodynamic alterations in hypertensive patients at rest and during passive head-up tilt. J Hypertens 2013; 31(5):906915.

[18] Hanninen MR, Niiranen TJ, Puukka PJ, et al. Determinants of masked hypertension in the general population: the Finn-Home study. J Hypertens 2011;29(10):1880-1888.

[19] Lindroos AS, Jula AM, Puukka PJ, et al. Comparison of acceptability of traditional and novel blood pressure measurement methods. Am J Hypertens 2016;29(6):679-683.

[20] Howie BM, Donnelly P, Marchini J. A flexible and accurate genotype imputation method for the next generation genome-wide association studies. PloS Genet 2009;5(6):e1000529.

[21] Patterson N, Price AL, Reich D. Population structure and eigenanalysis. PloS Genet 2006;2(12):e190.

[22] Price AL, Weale ME, Patterson N, et al. Long-range LD can confound genome scans in admixed populations. Am J Hum Genet 2008;83(1):132-135.

[23] Purcell S, Neale B, Todd-Brown K, et al. PLINK: a tool set for whole-genome association and population-based linkage analyses. Am J Hum Genet 2007;81(3):559-575.

[24] Willer CJ, Li Y, Abecasis GR. METAL: fast and efficient meta-analysis of genomewide association scans. Bioinformatics 2010;26(17):2190-2191.

[25] McLaren W, Gil L, Hunt SE, et al. The Ensembl variant effect predictor. Genome Biol 2016;17(1):122.

[26] Variant Effect Predictor (VEP) [Internet]. Hinxton, Cambridgeshire, UK: The European Bioinformatics Institute (EMBL-EBI); [cited 2018 Jun 12]. Available from http://www.ensembl.org/info/docs/tools/vep/index.html 
[27] Aken BL, Ayling S, Barrell D, et al. The Ensembl gene annotation system. Database 2016;baw093.

[28] Ensembl Genome Browser [Internet]. Hinxton, Cambridgeshire, UK: The European Bioinformatics Institute (EMBL-EBI); [cited 2018 Jun 12]. Available from http/www.emsebl.org

[29] GTEx Consortium, Laboratory, Data Analysis \& Coordinating Center (LDACC)-Analysis Working Group, Statistical Methdos groups-Analysis Working Group et al. Genetic effects on gene expression across human tissues. Nature 2017;550(7675):204-213.

[30] The Genotype-Tissue Expression (GTEx) Portal (v7) [Internet]. Cambridge, MA: The Broad Institute of MIT and Harvard; [cited 2019 Feb 10]. Available from http://gtexportal.org [31] UK Biobank GWAS by Neale lab [Internet]. Analytical and Translation Genetics Unit, Massachusetts General Hospital, Boston, MA: Neale B; [cited 2018 Jun 8]. Available from: http://www.nealelab.is

[32] Genetics of Personality Consortium, de Moor MH, van den Berg, et al. Meta-analysis of genome-wide association studies for neuroticism and the polygenic association with major depressive disorder. JAMA Psychiatry 2015;72(7):642-650.

[33] Okbay A, Baselmans BM, De Neve JE, et al. Genetic variants associated with subjective wellbeing, depressive symptoms, and neuroticism identified through genome-wide analyses. Nat Genet 2016;48(6):624-633.

[34] International Consortium for Blood Pressure Genome-Wide Association Studies, Ehret GB, Munroe PB, et al. Genetic variants in novel pathways influence blood pressure and cardiovascular disease risk. Nature 2011;478(7367):103-109.

[35] Staley JR, Blackshaw J, Kamat MA, et al. PhenoScanner: a database of human genotypephenotype associations. Bioinformatics 2016;32(20):3207-3209.

[36] Phenoscanner (v1.1) - A database of human genotype-phenotype associations [Internet]. Cambridge, UK: University of Cambridge; [cited 2018 Jun 8]. Available from http://www.phenoscanner.medschl.cam.ac.uk/phenoscanner

[37] Hermani G, Zheng J, Elsworth B, et al. The MR-Base platform supports systematic causal inference across the human phenome. eLife 2018;7:e34408.

[38] MR-Base PheWAS [Intenet]. Bristol, UK: University of Bristol; [cited 2018 Jun 8]. Available from http://phewas.mrbase.org/

[39] Schunkert H, Konig IR, Kathiresan S, et al. Large-scale association analysis identifies 13 new susceptibility loci for coronary artery disease. Nat Genet 2011;43(4):333-338. 
[40] Patron M, Sprenger HG, Langer T. m-AAA proteases, mitochondrial calcium homeostasis and neurodegeneration. Cell Res 2018;28(3):296-306.

[41] Almontashiri NA, Chen HH, Mailloux RJ, et al. SPG7 variant escapes phosporylationregulated processing by AFG3L2, elevates mitochondrial ROS, and is associated with multiple clinical phenotypes. Cell Rep 2014;7(3):834-847.

[42] Ferreira LR, Abrantes EF, Rodrigues CV, et al. Identification and characterization of a novel mouse gene encoding a Ras-associated guanine nucleotide exchange factor: expression in macrophages and myocarditis elicited by Trypanosoma cruzi parasites. J Leukoc Biol 2002;72(6):1215-1227.

[43] Ng WL, Marinov GK, Liau ES, et al. Inducible RasGEF1B circular RNA is a positive regulator of ICAM-1 in the TLR4/LPS pathway. RNA Biol 2016;13(9):861-871.

[44] Randall JC, Winkler TW, Kutalik Z, et al. Sex-stratified genome-wide association studies including 270,000 individuals show sexual dimorphism in genetic loci for anthropometric traits. PloS Genet 2013;9(6):e1003500.

[45] Spruill TM, Pickering TG, Schwartz JE, et al. The impact of perceived hypertension status on anxiety and the white coat effect. Ann Behav Med 2007;34(1):1-9,

[46] Terracciano A, Scuteri A, Strait J, et al. Are personality traits associated with white-coat and masked hypertension? J Hypertens 2014;32(10):1987-1992; discussion 1992. 


\section{Table titles}

Table 1. Clinical characteristics of the discovery cohort (GENRES) and replication cohorts (DYNAMIC, Finn-Home and DILGOM).

Table 2. Top SNPs associated with systolic white-coat effect in GENRES and replication in DYNAMIC, Finn-Home and DILGOM.

Table 3. Replication analysis of top SNPs associated with systolic white-coat effect in GENRES in men only.

Table 4. Top SNPs associated with diastolic white-coat effect in GENRES and replication in DYNAMIC, Finn-Home and DILGOM.

Table 5. Replication analysis of top SNPs associated with diastolic white-coat effect in GENRES in men only. 


\section{Figure captions}

Figure 1. Manhattan plots of the association $p$ values from the discovery genome-wide association study in GENRES. (A) systolic white-coat effect (B) diastolic white-coat effect. The y-axis shows the $-\log _{10}(p$ values) of each genotyped SNP and the $\mathrm{x}$-axis shows their chromosomal position. The horizontal lines correspond to genome wide $\left(5 \times 10^{-8}\right)$ and suggestive $\left(1 \times 10^{-5}\right) p$ value thresholds. SNPs above the suggestive threshold are annotated. Abbreviations: SNP, single nucleotide polymorphism.

A

Systolic white-coat effect

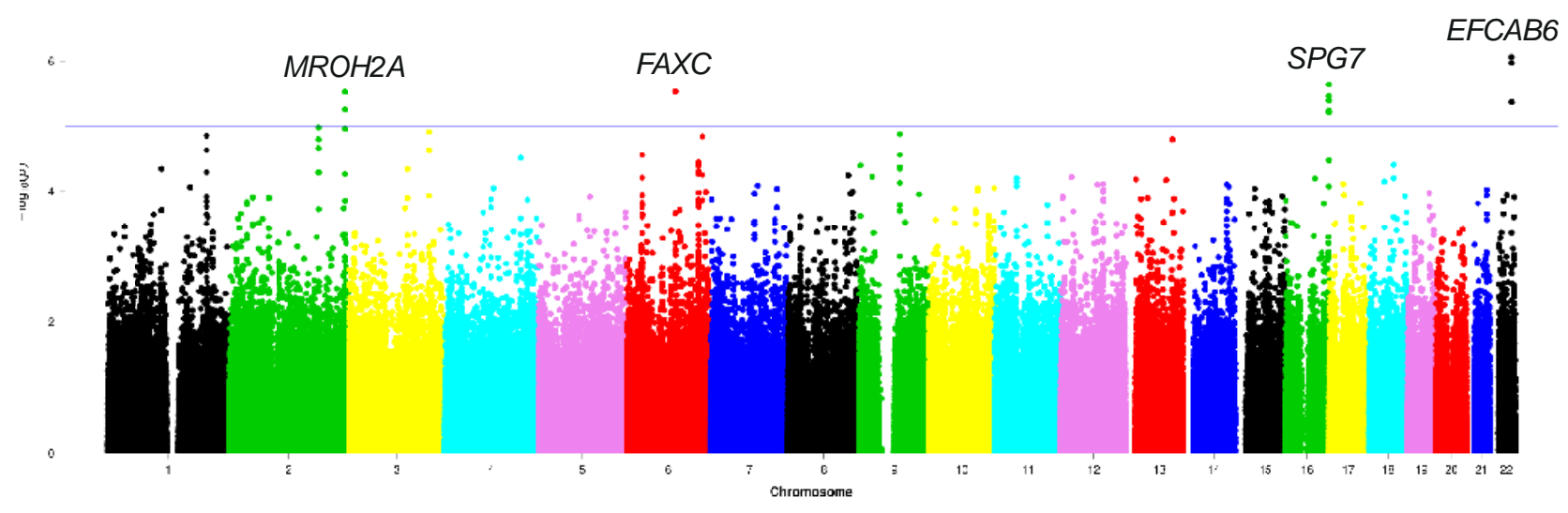

B

Diastolic white-coat effect

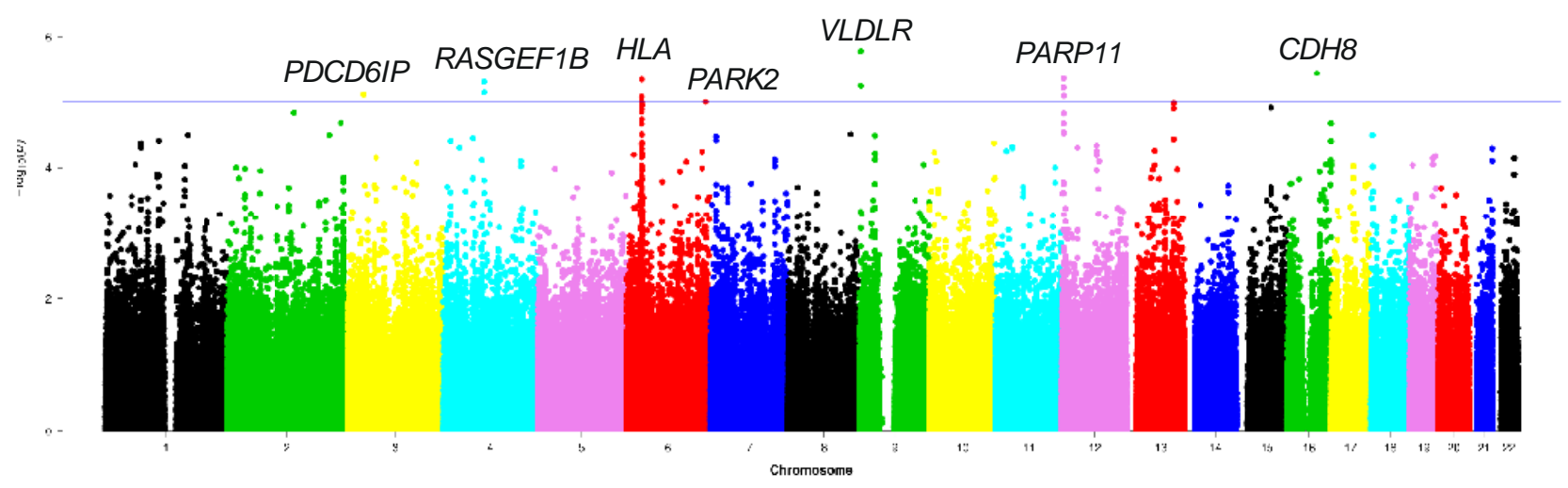


TABLE 1 Clinical characteristics of the discovery cohort (GENRES) and replication cohorts (DYNAMIC, Finn-Home and DILGOM).

\begin{tabular}{lcccc}
\hline Parameter & GENRES & DYNAMIC & Finn-Home & DILGOM \\
\hline$n$ & 206 & 182 & 773 & 182 \\
Age (years) & $50.8 \pm 6.3$ & $47.5 \pm 11.7$ & $55.6 \pm 7.9$ & $56.3 \pm 11.3$ \\
Men & $100 \%$ & $60 \%$ & $48 \%$ & $47 \%$ \\
Body Mass Index $\left(\mathrm{kgm}^{-2}\right)$ & $26.5 \pm 2.7$ & $27.0 \pm 4.1$ & $27.3 \pm 3.9$ & $26.6 \pm 4.4$ \\
Antihypertensive use & - & - & $21 \%$ & $29 \%$ \\
Smoking & $16 \%$ & $15 \%$ & $22 \%$ & $8 \%$
\end{tabular}

Blood pressure levels

Office measurement

$\begin{array}{lcccc}\mathrm{SBP}(\mathrm{mmHg}) & 152 \pm 13 & 145 \pm 18 & 137 \pm 19 & 129 \pm 18 \\ \mathrm{DBP}(\mathrm{mmHg}) & 100 \pm 7 & 92 \pm 11 & 84 \pm 8 & 79 \pm 10\end{array}$

Ambulatory measurement

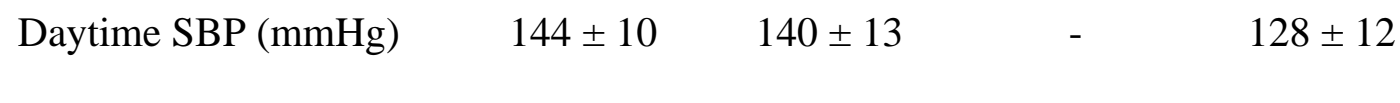

$\begin{array}{lllll}\text { Daytime DBP }(\mathrm{mmHg}) & 99 \pm 6 & 88 \pm 8 & - & 78 \pm 8\end{array}$

\section{Home measurement}

$\begin{array}{lllc}\mathrm{SBP}(\mathrm{mmHg}) & - & - & 129 \pm 17 \\ \mathrm{DBP}(\mathrm{mmHg}) & - & - & 81 \pm 9\end{array}$

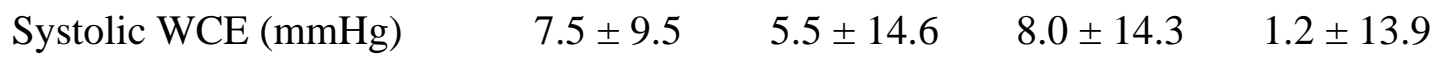

Diastolic WCE $(\mathrm{mmHg}) \quad 0.3 \pm 5.6 \quad 4.0 \pm 8.6 \quad 3.6 \pm 7.5 \quad 0.3 \pm 8.5$

Values are presented as mean \pm standard deviation.

Abbreviations: SBP, systolic white-coat effect; DBP, diastolic white-coat effect; WCE, white-coat effect. 
1 TABLE 2 Top SNPs associated with systolic white-coat effect in GENRES and replication in DYNAMIC, Finn-Home and DILGOM

\begin{tabular}{|c|c|c|c|c|c|c|c|c|c|c|c|c|c|c|}
\hline \multicolumn{9}{|c|}{$\begin{array}{l}\text { GENRES } \\
(n=206)\end{array}$} & \multicolumn{2}{|c|}{$\begin{array}{c}\text { DYNAMIC } \\
(n=182)\end{array}$} & \multicolumn{2}{|c|}{$\begin{array}{l}\text { Finn-Home } \\
(n=773)\end{array}$} & \multicolumn{2}{|c|}{$\begin{array}{l}\text { DILGOM } \\
(n=182)\end{array}$} \\
\hline Locus & rsID & $\begin{array}{l}\text { Chr:Pos } \\
\text { (build 37) }\end{array}$ & Gene & EA & EAF & $\beta$ & SE & $p$ & $\beta$ & $p$ & $\beta$ & $p$ & $\beta$ & $p$ \\
\hline 1 & rs9614176 & 22:43922499 & EFCAB6 & A & 0.14 & 5.4 & 1.1 & $8.7 \times 10^{-7}$ & -1.7 & $(0.5)$ & -0.8 & $(0.4)$ & -1.1 & $(0.5)$ \\
\hline 2 & rs2292954 & $16: 89613123$ & $S P G 7$ & $\mathrm{G}$ & 0.10 & 6.2 & 1.3 & $2.3 \times 10^{-6}$ & 2.2 & 0.4 & 2.4 & $0.03 *$ & -3.5 & $(0.05)$ \\
\hline 3 & rs4454147 & $6: 99575874$ & $F A X C$ & G & 0.48 & -3.7 & 0.8 & $2.9 \times 10^{-6}$ & 0.1 & $(1.0)$ & -0.2 & 0.8 & 1.0 & $(0.5)$ \\
\hline 4 & rs7606893 & $2: 234732816$ & $M R O H 2 A$ & A & 0.49 & 3.6 & 0.8 & $3.0 \times 10^{-6}$ & -0.3 & $(0.9)$ & -0.5 & $(0.5)$ & -0.9 & $(0.5)$ \\
\hline
\end{tabular}

SNPs with $p<1 \times 10^{-5}$ in the discovery GWAS in GENRES are shown in the order of significance.

The effect $(\beta)$ of each SNP on white-coat effect is presented as mmHg.

$*$ indicates suggestive replication with same direction of effect.

$p$ values are presented in parentheses if the direction of effect is different from the discovery GWAS.

Abbreviations: SNP, single nucleotide polymorphism; Chr, chromosome; Pos, position; EA, effect allele; EAF, effect allele frequency, SE,

standard error. 
8 TABLE 3 Replication analysis of top SNPs associated with systolic white-coat effect in GENRES in men only.

\begin{tabular}{|c|c|c|c|c|c|c|c|c|c|c|c|c|c|}
\hline \multirow[b]{3}{*}{ Locus } & \multicolumn{2}{|c|}{$\begin{array}{l}\text { GENRES } \\
(n=206)\end{array}$} & & & \multicolumn{3}{|c|}{$\begin{array}{c}\text { DYNAMIC } \\
(n=109)\end{array}$} & \multicolumn{3}{|c|}{$\begin{array}{c}\text { Finn Home } \\
(n=367)\end{array}$} & \multicolumn{3}{|c|}{$\begin{array}{c}\text { DILGOM } \\
(n=85)\end{array}$} \\
\hline & \multicolumn{4}{|c|}{ WCE } & \multirow[b]{2}{*}{$p_{(\operatorname{sex} \times S N P)}$} & \multicolumn{2}{|c|}{$\underline{\mathrm{WCE}}$} & \multicolumn{3}{|c|}{$\underline{\mathrm{WCE}}$} & \multirow[b]{2}{*}{$p_{(\operatorname{sex} \times S N P)}$} & \multicolumn{2}{|c|}{$\underline{\mathrm{WCE}}$} \\
\hline & rsID & EA & $\beta$ & $p$ & & $\beta$ & $p$ & $p_{(\operatorname{sex} \times S N P)}$ & $\beta$ & $p$ & & $\beta$ & $p$ \\
\hline 1 & rs9614176 & A & 5.4 & $8.7 \times 10^{-7}$ & 0.8 & - & - & 0.3 & - & - & 0.6 & - & - \\
\hline 2 & rs2292954 & G & 6.2 & $2.3 \times 10^{-6}$ & 0.7 & - & - & 0.2 & - & - & 1.0 & - & - \\
\hline 3 & rs4454147 & G & -3.7 & $2.9 \times 10^{-6}$ & 0.3 & 1.2 & $(0.5)$ & $0.04 *$ & -1.6 & 0.07 & 0.2 & 3.0 & $(0.12)$ \\
\hline 4 & rs7606893 & A & 3.6 & $3.0 \times 10^{-6}$ & 0.2 & - & - & 0.2 & - & - & 0.8 & - & - \\
\hline
\end{tabular}

Separate replication analysis was conducted in men only if $\operatorname{sex} \times \mathrm{SNP}$ interaction $p$ value was $<0.10$ in any of the replication cohorts

The effect $(\beta)$ of each SNP on white-coat effect is presented as $\mathrm{mmHg}$.

$*$ indicates significant sex $\times$ SNP interaction.

$p$ values are presented in parentheses if the direction of effect is different from the discovery GWAS.

13 Abbreviations: SNP, single nucleotide polymorphism; EA, effect allele; WCE, white-coat effect 
TABLE 4 Top SNPs associated with diastolic white-coat effect in GENRES and replication in DYNAMIC, Finn-Home and DILGOM

\begin{tabular}{|c|c|c|c|c|c|c|c|c|c|c|c|c|c|c|}
\hline & & & $\begin{array}{l}\text { GENRE } \\
(n=206\end{array}$ & & & & & & $\begin{array}{r}\text { DYI } \\
(n\end{array}$ & $\begin{array}{l}\text { MIC } \\
82)\end{array}$ & $\begin{array}{r}\text { Finr } \\
(n\end{array}$ & $\begin{array}{l}\text { Home } \\
773)\end{array}$ & $\begin{array}{l}\text { DII } \\
(n=\end{array}$ & $\begin{array}{l}\text { OM } \\
182)\end{array}$ \\
\hline Locus & rsID & Chr:Pos & Gene & EA & EAF & $\beta$ & $\mathrm{SE}$ & $p$ & $\beta$ & $p$ & $\beta$ & $p$ & $\beta$ & $p$ \\
\hline 1 & rs10965522 & 9:2301692 & $V L D L R$ & A & 0.24 & 2.8 & 0.6 & $1.7 \times 10^{-6}$ & 0.4 & 0.7 & 0.0 & 1.0 & -0.5 & $(0.6)$ \\
\hline 2 & rs 2650673 & 16:61120981 & $\mathrm{CDH}$ & A & 0.44 & 2.2 & 0.5 & $3.6 \times 10^{-6}$ & 0.1 & 0.9 & 0.1 & 0.9 & -0.2 & $(0.8)$ \\
\hline 3 & rs11062980 & $12: 4150135$ & PARP11 & A & 0.44 & -2.3 & 0.5 & $4.4 \times 10^{-6}$ & 1.8 & $(0.06)$ & 0.2 & $(0.6)$ & -0.6 & 0.5 \\
\hline 4 & rs2064479 & $6: 33072240$ & $H L A-D P B 1$ & A & 0.28 & 2.4 & 0.5 & $4.5 \times 10^{-6}$ & 0.1 & 0.9 & 0.1 & 0.8 & -0.3 & $(0.8)$ \\
\hline 5 & rs10033652 & 4:82493067 & $R A S G E F 1 B$ & A & 0.49 & 2.2 & 0.5 & $4.9 \times 10^{-6}$ & 0.4 & 0.7 & -0.3 & $(0.4)$ & 1.8 & $0.04 *$ \\
\hline 6 & rs 2053425 & 3:34241686 & PDCD6IP & A & 0.25 & 2.3 & 0.5 & $7.7 \times 10^{-6}$ & 0.4 & 0.8 & -0.7 & $(0.11)$ & -1.4 & $(0.2)$ \\
\hline 7 & rs6921226 & $6: 162128543$ & PARK2 & G & 0.41 & -2.2 & 0.5 & $9.9 \times 10^{-6}$ & 1.0 & $(0.3)$ & 0.3 & $(0.4)$ & -0.4 & 0.7 \\
\hline
\end{tabular}

SNPs with $p<1 \times 10^{-5}$ in the discovery GWAS in GENRES are shown in the order of significance.

The effect $(\beta)$ of each SNP on white-coat effect is presented as $\mathrm{mmHg}$.

* indicates suggestive replication with same direction of effect.

$p$ values are presented in parentheses if the direction of effect is different from the discovery GWAS.

19 SNP, single nucleotide polymorphism; Chr, chromosome; Pos, position; EA, effect allele; EAF, effect allele frequency, SE, standard error. 
TABLE 5 Replication analysis of top SNPs associated with diastolic white-coat effect in GENRES in men only.

\begin{tabular}{|c|c|c|c|c|c|c|c|c|c|c|c|c|c|}
\hline \multicolumn{5}{|c|}{$\begin{array}{l}\text { GENRES } \\
(\mathrm{n}=206)\end{array}$} & \multicolumn{3}{|c|}{$\begin{array}{c}\text { DYNAMIC } \\
(\mathrm{n}=109)\end{array}$} & \multicolumn{3}{|c|}{$\begin{array}{l}\text { Finn Home } \\
(\mathrm{n}=367)\end{array}$} & \multicolumn{3}{|c|}{$\begin{array}{c}\text { DILGOM } \\
(\mathrm{n}=85)\end{array}$} \\
\hline \multirow{2}{*}{ Locus } & \multirow{2}{*}{ rsID } & \multirow{2}{*}{ EA } & \multicolumn{2}{|c|}{ WCE } & \multirow{2}{*}{$\mathrm{P}(\operatorname{sex} \times \mathrm{SNP})$} & \multicolumn{2}{|c|}{$\underline{\text { WCE }}$} & \multirow{2}{*}{$\mathrm{P}_{(\mathrm{sex} \times \mathrm{SNP})}$} & \multicolumn{2}{|c|}{ WCE } & \multirow{2}{*}{$\mathrm{P}_{(\mathrm{sex} \times \mathrm{SNP})}$} & \multicolumn{2}{|c|}{$\underline{\mathrm{WCE}}$} \\
\hline & & & $\beta$ & $\mathrm{P}$ & & $\beta$ & $\mathrm{P}$ & & $\beta$ & $\mathrm{P}$ & & $\beta$ & $\mathrm{P}$ \\
\hline 1 & rs 10965522 & A & 2.8 & $1.7 \times 10^{-6}$ & 0.2 & - & - & 0.8 & - & - & 0.5 & - & - \\
\hline 2 & rs2650673 & A & 2.2 & $3.6 \times 10^{-6}$ & 0.6 & -0.5 & (0.7) & 0.7 & -0.1 & $(0.9)$ & $0.097 *$ & 1.8 & 0.2 \\
\hline 3 & rs 11062980 & A & -2.3 & $4.4 \times 10^{-6}$ & $0.06 *$ & 0.8 & $(0.5)$ & 0.3 & -0.3 & 0.6 & 0.12 & -1.9 & 0.07 \\
\hline 4 & rs2064479 & A & 2.4 & $4.5 \times 10^{-6}$ & 0.6 & - & - & 0.8 & - & - & 0.4 & - & - \\
\hline 5 & rs 10033652 & A & 2.2 & $4.9 \times 10^{-6}$ & 0.2 & - & - & 0.9 & - & - & 0.9 & - & - \\
\hline 6 & rs2053425 & A & 2.3 & $7.7 \times 10^{-6}$ & 0.9 & - & - & 0.2 & - & - & 0.3 & - & - \\
\hline 7 & rs6921226 & G & -2.2 & $9.9 \times 10^{-6}$ & 0.6 & - & - & 0.9 & - & - & 0.6 & - & - \\
\hline
\end{tabular}

Separate replication analysis was conducted in men only if sex $\times$ SNP interaction $p$ value was $<0.10$ in any of the replication cohorts.

The effect $(\beta)$ of each SNP on white-coat effect is presented as $\mathrm{mmHg}$.

$*$ indicates significant $\operatorname{sex} \times \mathrm{SNP}$ interaction $(p<0.10)$.

$p$ values are presented in parentheses if the direction of effect is different from the discovery GWAS.

Abbreviations: SNP, single nucleotide polymorphism; EA, effect allele; WCE, white-coat effect. 
Supplementary Table 1: Covariates used to generate regression residuals for white-coat effect in GENRES

\begin{tabular}{l|c|c}
\hline Lifestyle factor & $\begin{array}{c}\text { Systolic WCE } \\
\beta / p\end{array}$ \\
\hline Ambulatory daytime SBP & $\beta / \mathrm{p}$ & - \\
Ambulatory daytime DBP & $-0.2 / 0.002$ & $-0.2 / 7.8 \times 10-4$ \\
Age normalized & - & $1.8 / 7.2 \times 10-7$ \\
Body mass index kgm-2 & $4.1 / 9.9 \times 10-11$ & $0.4 / 0.001$ \\
Smoking (1=no, 2=yes) & $0.1 / 0.13$ & $-1.3 / 0.189$ \\
Previous antihypertensive medication (1=no, 2=yes) & $-4.177 / 0.01$ & $\mathrm{~ns}$ \\
Waist-hip ratio & $\mathrm{ns}$ & $\mathrm{ns}$ \\
Serum Creatinine & $\mathrm{ns}$ & $\mathrm{ns}$ \\
dU-Na & $\mathrm{ns}$ & $\mathrm{ns}$ \\
r2 of the model & $\mathrm{ns}$ & 0,23 \\
Adjusted r2 of the model & 0,25 & 0,22 \\
\hline
\end{tabular}

A covariate was included in the models if association $p$ value was $<0.10$ for either systolic or diastolic WCE. Abbreviations: WCE, white-coat effect; SBP, systolic blood pressure; DBP, diastolic blood pressure 
Supplementary Figure 1. The GENRES Study design. The timings of the placebo OBP and ABP measurements used for the discovery GWAS are marked in the figure with black arrows and blue boxes. Abbreviations: OBP, office blood pressure; ABP, ambulatory blood pressure; ECG, electrocardiography.

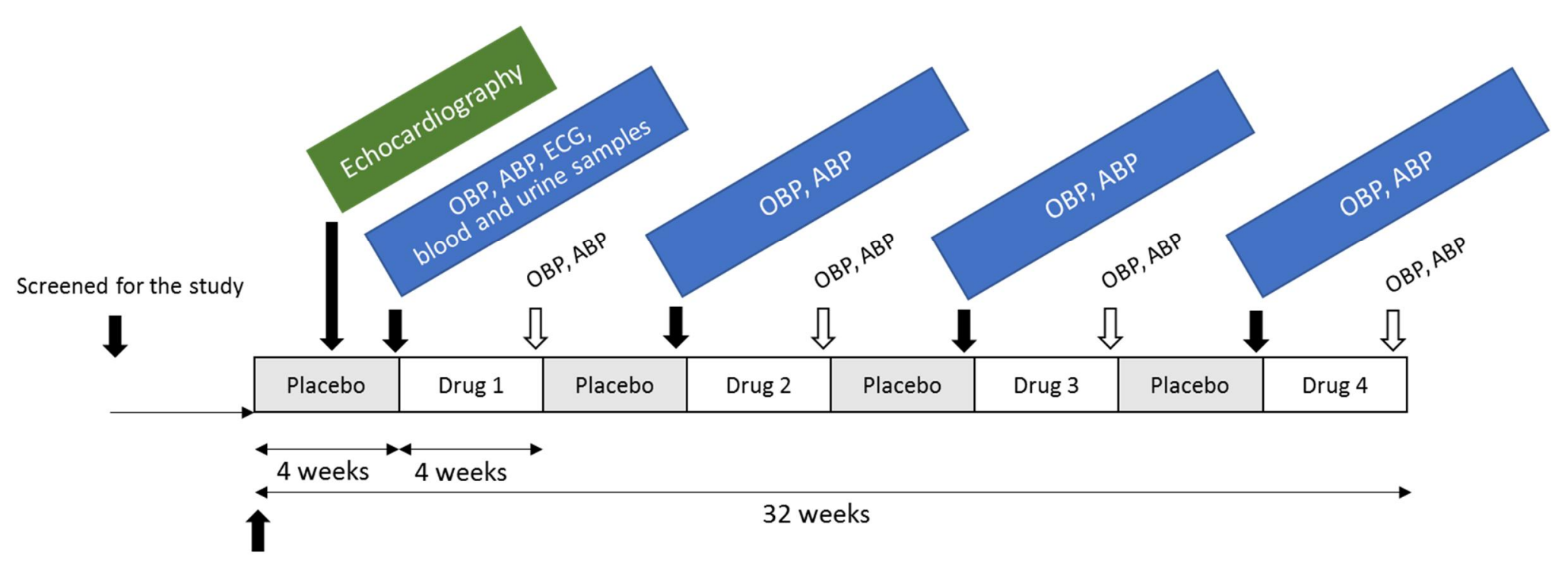

Prescribed

antihypertensive drugs discontinued 
Supplementary Figure 2. Quantile-quantile plots of the association results.

This figure shows the quantile-quantile plots created in $\mathrm{R}$ for two-sided $\mathrm{P}$ values of the genome-wide association analysis on (A) systolic white coat effect and (B) diastolic white coat effect. The $\mathrm{x}$-axis shows the expected distribution of $\neg \log _{10}(p$ values $)$ and the y-axis shows the observed $\log _{10}(p$ values $)$.

A

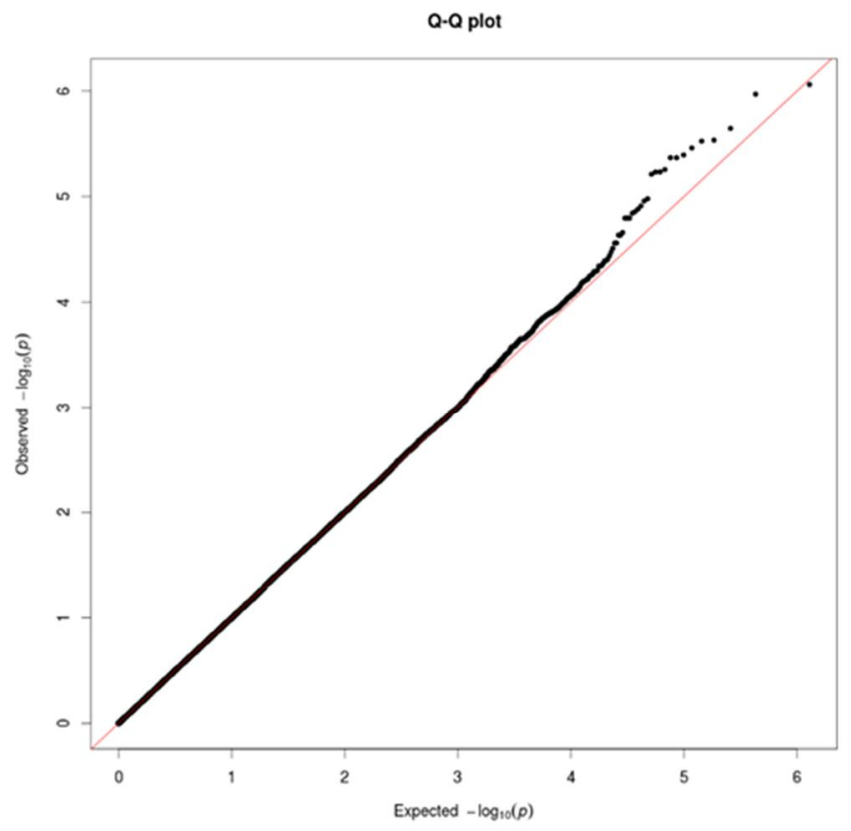

B

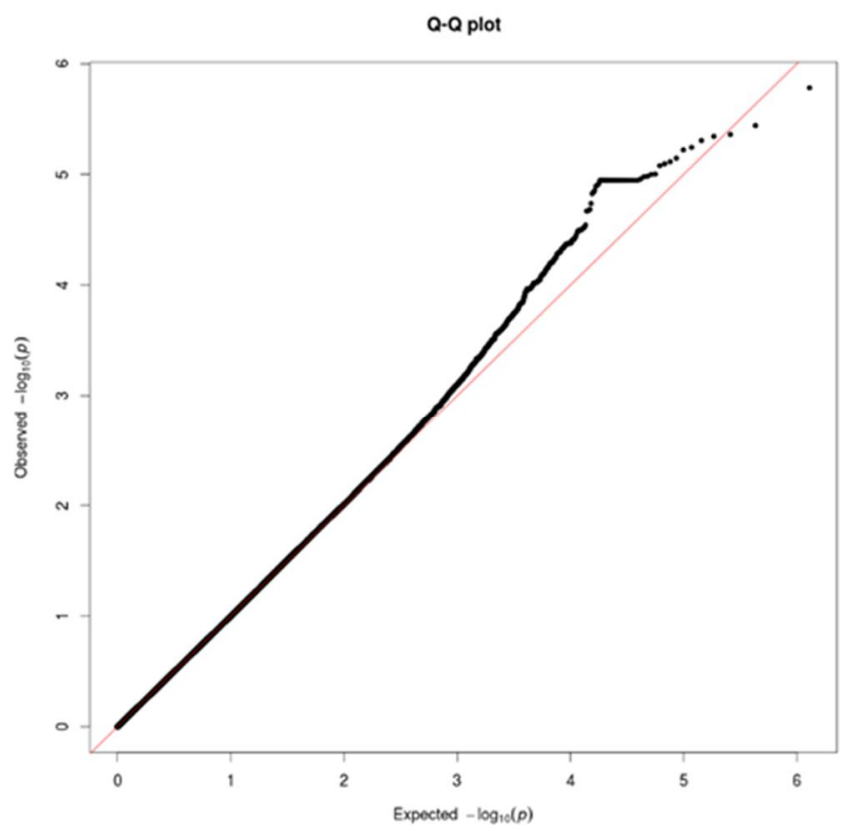


Supplementary Figure 3. LocusZoom plots of the top loci for systolic white coat effect in GENRES. This figure shows the regional Manhattan plots created with LocusZoom for the best loci for systolic WCE in the discovery cohort (GENRES). The y-axis shows the $-\log _{10}(p$ value) of each genotyped SNP and the xaxis shows their chromosomal position. Blue line depicts the recombination rate from HAPMAP(EU) population. The SNPs are coloured to reflect pairwise LD $\left(r^{2}\right)$ with the most significantly associated SNP in GENRES. Genes mapping to each locus are shown in the lower panel.

A

rs9614176

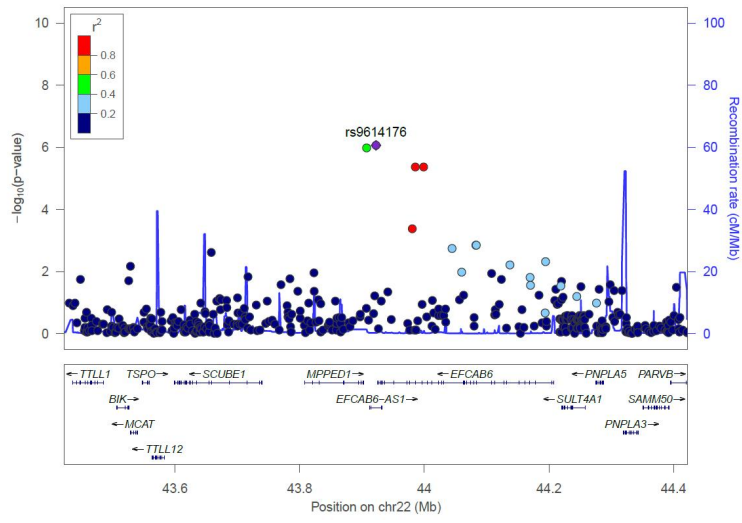

C

rs4454147

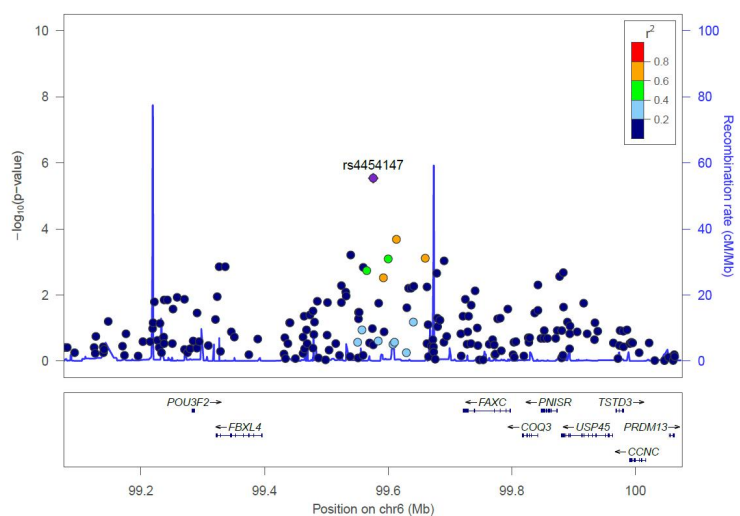

B

rs2292954

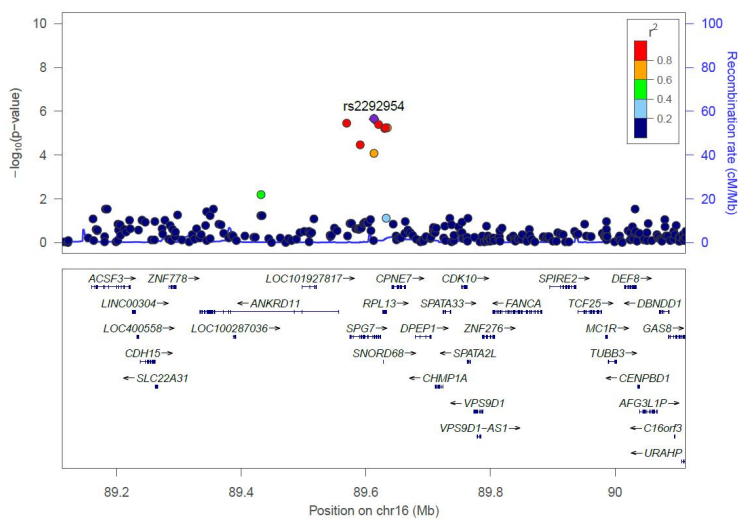

D

rs7606893

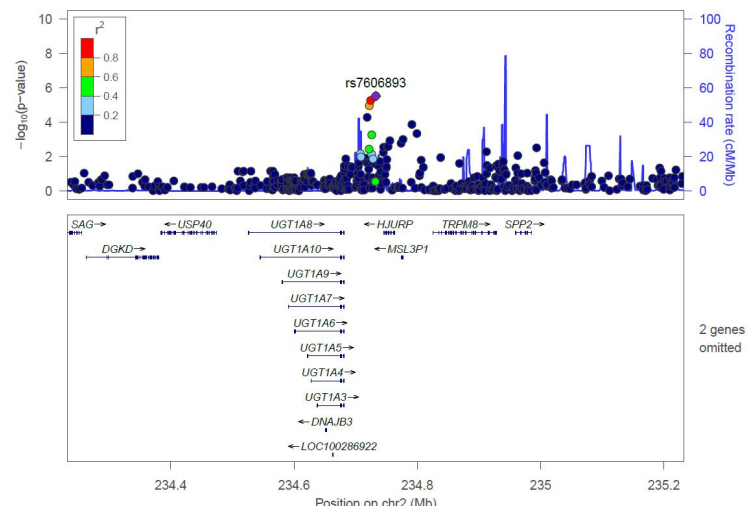


Supplementary Figure 4. LocusZoom plots of the top loci for diastolic white coat effect.

This figure shows the regional Manhattan plots created with LocusZoom for the best loci for systolic WCE in the discovery cohort (GENRES). The y-axis shows the $-\log _{10}(p$ value) of each genotyped SNP and the $\mathrm{x}-$ axis shows their chromosomal position. Blue line depicts the recombination rate from HAPMAP(EU) population. The SNPs are coloured to reflect pairwise LD $\left(r^{2}\right)$ with the most significantly associated SNP in GENRES. Genes mapping to each locus are shown in the lower panel.

A

rs10965522

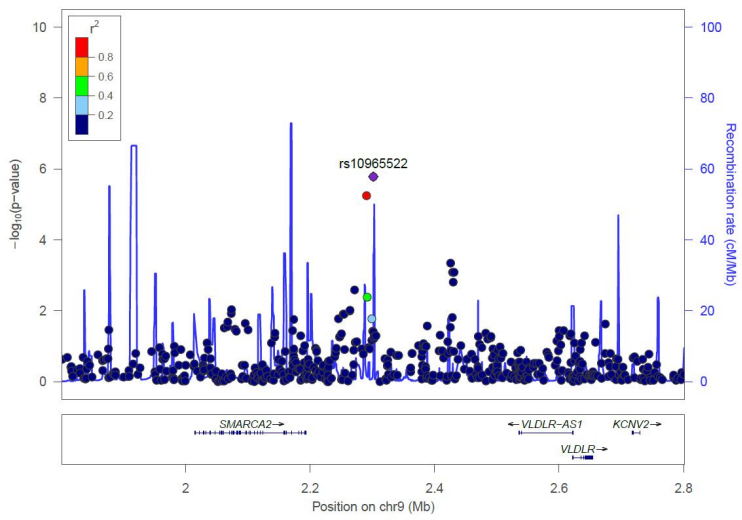

C

rs11062980

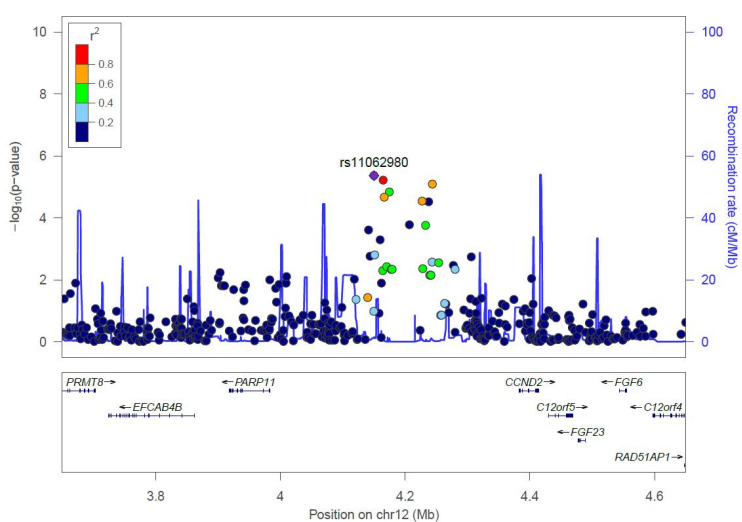

B

rs2650573

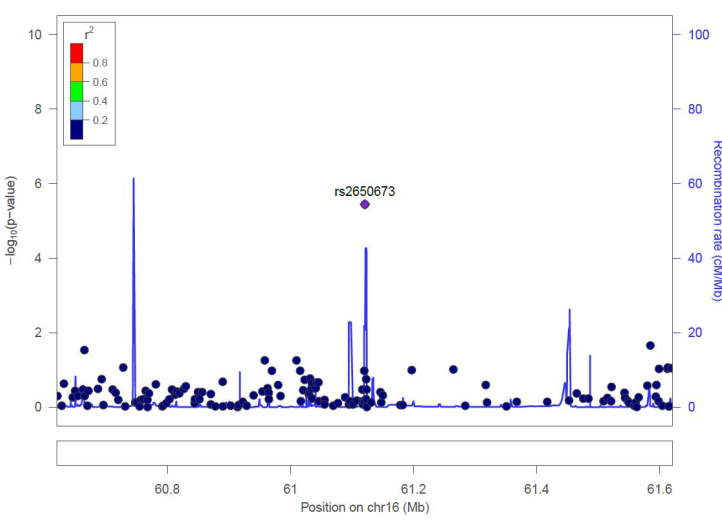

D

rs2064479

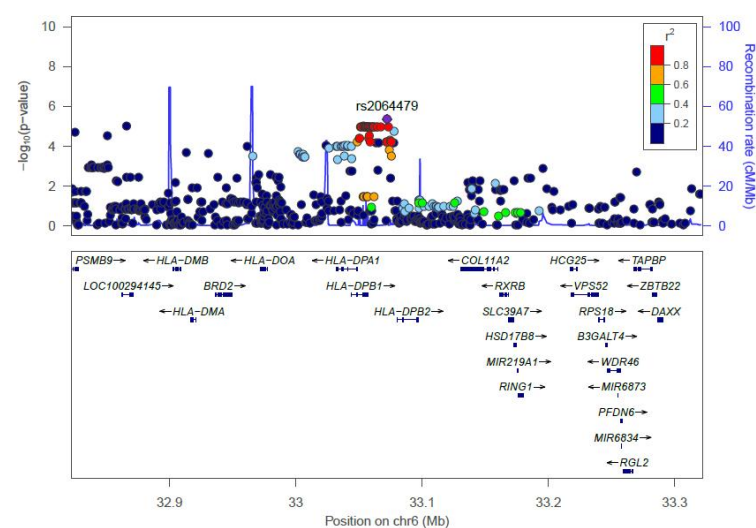


E

rs10033625

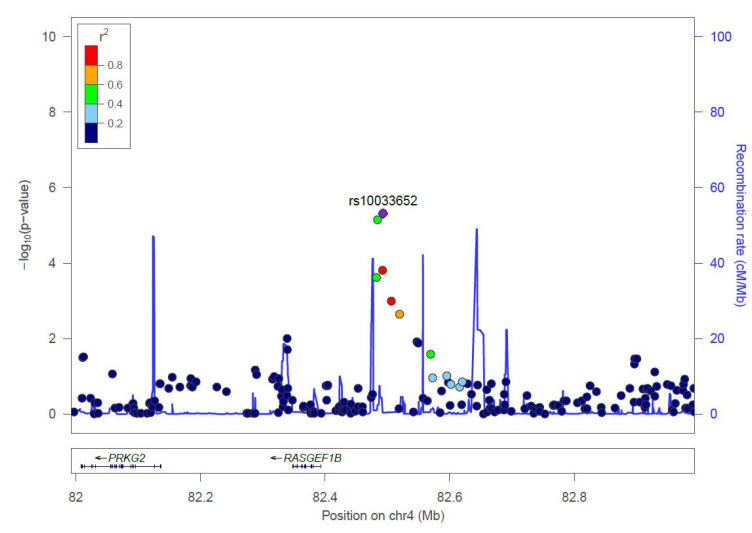

G

rs6921226

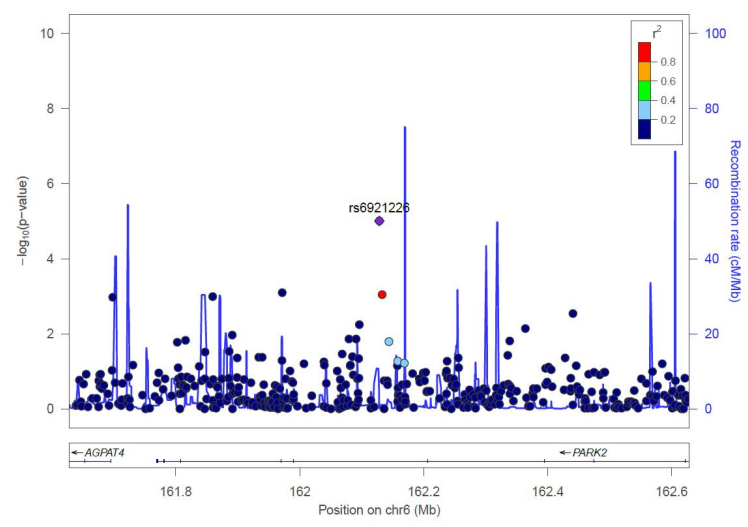

F

rs2053425

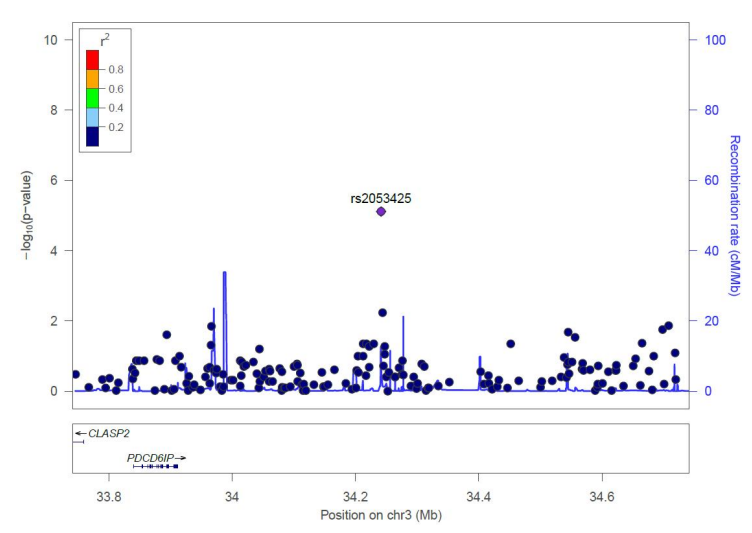

